\title{
La Rocea di Arquata del Tronto: simbolo di rinascita per il territorio marchigiano colpito dal sisma
}

The fortress of Arquata del Tronto: symbol of rebirth for the Marche Region affected by the earthquake

\author{
Enrica Petrucci ${ }^{a}$, Diana Lapucci ${ }^{\text {b }}$, Noemi Lapucci ${ }^{c}$ \\ Universita di Camerino, Scuola di Architettura e Design, Ascoli Piceno, Italy \\ a enrica.petrucci@unicam.it; ${ }^{\text {b }}$ diana.lapucci@unicam.it; ${ }^{\mathrm{c}}$ noemi.lapucci@unicam.it
}

\begin{abstract}
The Fortress of Arquata del Tronto in the Marche region is an imposing structure that rises on the crag to the north of the historic center. It represents a typical example of military architecture in the Apennines Area. Its foundation is between the eleventh and the twelfth century, an initial phase of embankment which undergoes a gradual and progressive increase in structures to enhance the functionality of the fortress. The city of Ascoli Piceno, worried about consolidating the defense outposts located at its borders, soon took possession of it. From the thirteenth to the sixteenth century, the Rocca lived alternate events, clashing with nearby castles, especially with Norcia. At the end of the eighteenth century, Arquata will be absorbed in the territory of the Clitunno Department, in the ancient Duchy of Spoleto. During this period, it was partially restored, to house a stable military garrison, becoming the third fortress of the Trasimeno Department, until it returned under the Papal States. Continuous interventions make the structure suitable for military uses. At the end of the nineteenth century, Giuseppe Sacconi, as director of the Conservation Office in the Marche and Umbria Region, undertook an important restoration. The ruins of the fortress were reconfigured according to medieval forms, taken by analogy from the repertoire of fortifications in the Apennine area. A further conservative intervention was carried out in 1990 to allow a new use. Unfortunately, the seismic events in 2016-2017 have compromised the Roc$c a$, with large collapses that currently make the complex unusable. The intention is to undertake new restoration work, setting up a school construction site; this could represent a virtuous example to favor the rebirth of Arquata del Tronto, so strongly hit by the earthquake, through a project for the enhancement of its architectural heritage.
\end{abstract}

Keywords: Fortifications, earthquake, enhancement.

\section{Introduzione}

La rocca di Arquata è posta su un'altura che sovrasta l'alta valle del Tronto e domina su molte frazioni che rientrano nell'ambito di competenza del comune di Arquata del Tronto; la spina dorsale di questo territorio è rappresentata dalla strada consolare Salaria che lo collega a Roma; da essa si snodano anche ulteriori percorsi che conducono a sud verso la città dell'Aquila, dopo aver oltrepassato il centro di Amatrice e a ovest si può raggiungere l'Umbria superando le montagne appenniniche, in particolare la catena dei Sibillini. Fra le prime località umbre, s'incontra 
Norcia che ha combattuto per il dominio del territorio arquatano, contro la vicina Ascoli. Attualmente, la situazione di questi territori è particolarmente complessa causa de danni causati dagli eventi sismici 2016-2017. Il presente contributo si concentra sullo studio della fortificazione medievale, analizzata attraverso le modificazioni intercorse nel tempo, per definire una strategia di valorizzare, sia in fase di cantiere sia alla conclusione dei lavori restauro.

\section{Un breve regesto storico della rocca di Ar- quata del Tronto}

Le fortificazioni sono da sempre state soggette a continue evoluzioni e trasformazioni, dovute sia agli adattamenti alle tecniche belliche, sia ai diversi mutamenti socio-economici. Il termine architettura fortificata pertanto va inteso in una accezione molto ampia, che spazia tra strutture architettoniche anche funzionalmente molto diverse tra loro. In Italia, le fortificazioni sono diffuse su tutta la penisola e molte di queste sono sopravvissute fino ad oggi, in diversi stati di conservazione. Fra queste l'interessante rocca di Arquata del Tronto.

L'origine di questo insediamento militare è collocabile tra l'XI e il XII secolo, epoca in cui fu avviata la costruzione delle opere di fortificazione sul colle che sovrastava il piccolo insediamento di Arquata del Tonto. A questa prima fase di incastellamento seguì un graduale e progressivo incremento di nuove strutture, elevate nei secoli successivi, al fine di potenziare le funzionalità del presidio. Nel corso del XII secolo, come risulta dal Regestum Farfense, l'abate Berardo III, acquistò il contado e la Rocca Arquatam adquisivit et roccam de Cupulo (Amoni, 1999, p. 24).

L'imperatore Enrico V di Sassonia, mediante un diploma ne confermò il possesso all'abbazia reatina. Nei primi anni del XIII secolo ebbe inizio il fiorire e lo sviluppo del borgo che si dichiarò libero comune (Mauro, 1992, vol. I, pp. 313-356). Nel 1255, la Rocca era soggetta al dominio del governo ascolano. In quell'anno papa Alessandro IV ordina al Rettore della Marca di stanziare una somma da destinare alle casse della città, al fine di potenziare le strutture di difesa dei castel- li sparsi nel territorio di competenza, tra i quali Arquata. Nel 1293, Arquata, ed altri comuni della valle del Tronto, consolidarono i patti di fedeltà con Ascoli (Andreantonelli, 2007, p. 242). Dopo la scomparsa di Federico II, la città di Ascoli si era preoccupata di consolidare gli avamposti di difesa dislocati ai confini del suo vasto territorio, minacciati dalle aspirazioni di Manfredi di Sicilia, figlio del re svevo. Per queste ragioni la città era stata costretta "a fabricar negli Appennini un Forte per guardia dei confini affin di cautelarsi dalle scorrerie dei nemici" (Carfagna, 1996, pp. 59-63).

Nel XIV secolo la città di Ascoli, per non perdere il possedimento della Rocca e per sedare la ribellione di Arquata al suo dominio, cinse d'assedio il presidio e lo riconquistò, riconfermando anche il patto di alleanza. Nel 1350, Galeotto I Malatesta attaccò Arquata per sedarne la ribellione, ma riportò una solenne sconfitta a testimonianza della validità della struttura fortificata. Nel 1386, Arquata, strinse un patto con Fermo, promettendo la consegna di un Palio di seta per le festività dell'Assunta. Il paese era obbligato ad esborsare una notevole somma in cambio della protezione armata accordata dai Fermani. Tali forme asimmetriche di negoziati evidenziavano i tentativi fermani di estendere la propria sfera di influenza territoriale verso sud. Il dato di fondo che permane fino alle soglie del Quattrocento, relativamente ai quadri e alle dinamiche territoriali nel Piceno, è quello di una scarsa uniformità e dell'incapacità dei centri cittadini (Fermo ed Ascoli) di porsi come unici soggetti capaci di conferire stabilità agli assetti geo-politici di quest'area (Pirani, 2010, pp. 2122).

Alla fine del '300 Arquata è conquistata dalle truppe di Matteo d'Acquaviva e dal 1397 iniziano aspre battaglie tra Ascoli e Norcia. Arquata diviene una roccaforte di Norcia e dei ghibellini ascolani fuoriusciti. In seguito, la città di Ascoli la assedia nuovamente e riesce a riconquistarla. Dal XIII al XVI secolo, Arquata e la sua rocca vivono alterne vicissitudini, fatte di guerre repentine e lunghi conflitti con gli altri castelli vicini. Nel 1418, la città di Norcia chiese a papa Martino V la concessione del dominio di Arquata. Il pontefice, espresso il suo rifiuto, affidò al 
Vice-legato della Marca, il mandato di porre pace tra nursini e ascolani (Santis, 1999, vol. II, pp. 370- 374). Nel 1429, la fortezza torna sotto il controllo di Norcia, come riportato nella bolla pontificia, del 19 luglio, di papa Martino V. Fino alla metà del XV secolo, rimane in possesso della città umbra, come riferito da una bolla di papa Eugenio IV in cui i norcini si impegnano a corrispondere alla Camera Apostolica 230 fiorini annui pro taleis ed affictibus della fortezza. Il documento reca anche le due quietanze di rinnovo per gli anni 1454 e 1461. Il 16 gennaio 1466, il comandante Vincenzo Ficcadenti, con seicento uomini al seguito, espugna la Rocca, ponendo fine alla guerra tra Ascoli e Norcia (Fabiani, 1950, vol. II, pp. 109-114). Paolo II raggiunse l'accordo per una tregua quinquennale, sancita dalla bolla papale del 3 maggio 1466, sottoscritta dagli ascolani nell'agosto 1467. Durante la dominazione francese, Arquata è inserita nel territorio del Dipartimento del Clitunno che aveva per capoluogo la città di Spoleto e la sua Rocca, ritenuta ancora di importanza strategica, viene parzialmente restaurata, dotata di casematte e piazzole d'artiglieria, per ospitare un presidio militare permanente. La caduta del regime repubblicano conduce al ripristino delle istituzioni pontificie. Arquata è inserita nel II Mandamento di Ascoli (Luzi, 1889, pp. 125-126). Nel 1860 il territorio è annesso al Regno d'Italia: la sua fortezza perde definitivamente la sua importanza strategica e l'intero borgo si avvia verso una fase di lento declino (Fig. 1).

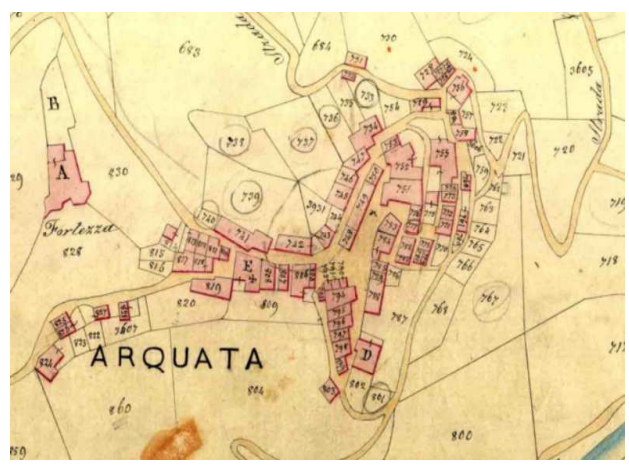

Fig. 1. Archivio di Stato di Ascoli Piceno, Fondo Catasti storici, Catasto Pio-Gregoriano del $1820 \mathrm{c}$.
Alcuni documenti d'archivio, citati da vari autori riportano le attrezzature, le armi e i pezzi di artiglieria a tiro parabolico di cui era dotata la guarnigione della Rocca di Arquata. In un inventario della fortezza comparivano, datato XV secolo: "una bombarda longa due pezzi fornita et ferrata con cippe, quatro piastre et zeppe, una spingarda longa ovver ciarabactana de doi pezzi fornita con lu cippo et cavallicto, una bombarda mezza de uno pezo co lu cippo, ferrata con una piastra, et zeppa de ferro et cippo fornita, una bombarda grossa senza cippo con piastre quatro et altre bombarde e bombardelle" (Galiè, Vecchioni, 2006, p. 83). In un altro documento, redatto al tempo in cui era castellano della Rocca Ambrogio da Montefortino, si trovano descritte numerose armi, mobili contenitori, botti per la conservazione del vino, utensili di uso quotidiano ed altri oggetti custoditi tra le mura della fortezza, tra i quali figuravano: "doimila e octocento aste senza ferri tra i targoni dipinti de l'armi de Papa Pio IV e la bandera de Papa Paulo II.”, ed ancora: "l'arca vecchia per fare lo pane, gli arconi granarii da tenere lo grano fra le pallocte del plumbo et scoppitti fra altre botti da octi some et corazze et celate et la robusta catena de ferro per levare il ponte levatoio et mucchi a piramide di palle de pietra per le bombarde e le mille e novecento aste guarnite de ferro et i tremila verrectoni senza aste" (Girolami, 1987, pp. 43-44).

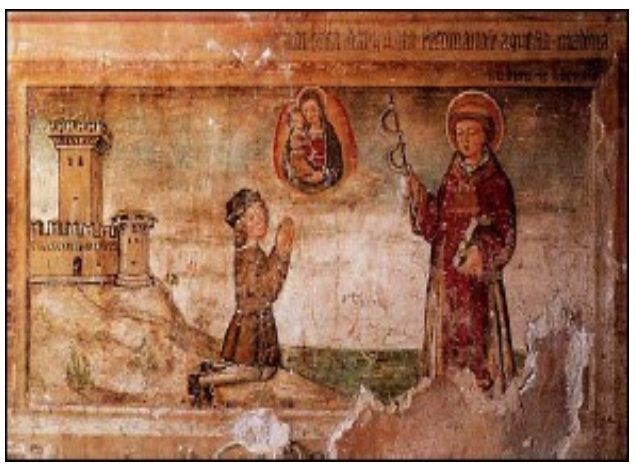

Fig. 2. Ex Voto posto nella fascia inferiore dell'abside nel Santuario dell'Icona Passatora di Amatrice. Nel dipinto sono rappresentati la Madonna e San Leonardo mentre liberano un prigioniero della fortezza arquatana che invoca la loro intercessione. 
La rocca di Arquata per la sua posizione strategica fu coinvolta nella "Prima guerra di Castro" che scaturì dai contrasti fra Odoardo Farnese, duca di Parma, Piacenza e Ronciglione, e la famiglia Barberini. Gli occasionali contrasti verificatisi tra membri delle due casate e soprattutto il deciso proposito del pontefice Urbano VIII di sottrarre ad Odoardo il ducato di Castro e dei cui privilegi la famiglia Farnese godeva da alcuni secoli portarono inevitabilmente al conflitto, scoppiato nel 1641 e protrattosi fino al 1644. La cronologia degli eventi può essere ricostruita dalle notizie desunte dalla corrispondenza barberiniana custodita nella Biblioteca Apostolica Vaticana, dove è raccolto il carteggio relativo all'attività del cardinal nipote Francesco Barberini. Dai carteggi è evidente l'importanza di un riordino di tutte le fortificazioni con opere dettate dall'urgenza di proteggere al meglio le zone strategiche.

La rocca di Arquata si presentava sufficientemente organizzata per la difesa, essendo presenti un doppio sistema di mura con corte interna, un'alta torre a est e un sistema di due rivellini sul lato sud-ovest (Fig. 3). Le lettere fanno riferimento alla distribuzione interna degli ambienti destinati alle varie funzioni, anche quelle residenziali.

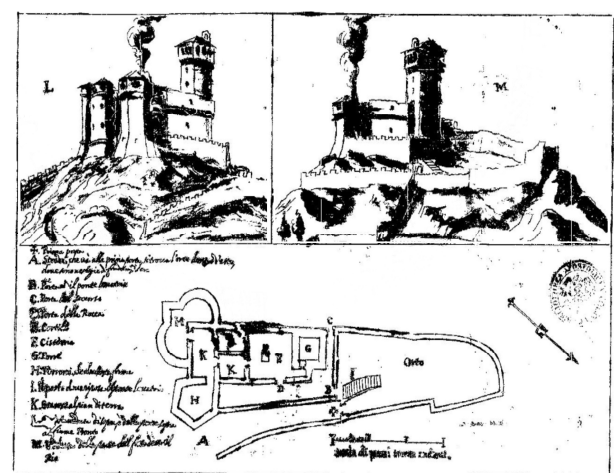

Fig. 3. Biblioteca Apostolica Vaticana, Barb Lat. 9901, f. 6r, Disegno della Rocca d'Arquata del Tronto.

Alla storia di questa fortezza è legata anche una tradizione leggendaria secondo la quale vi avrebbe soggiornato la regina Giovanna II d'Angiò, negli anni compresi tra il 1420 ed il 1435. In occasione di questo soggiorno, gli sto- rici riferiscono di alcuni interventi di restauro (Bucciarelli, 1982, pp. 13-16).

Nel primi anni del ' 900 , la fortificazione persa la sua originaria funzione di presidio difensivo, era stata abbandonata e versava in stato di avanzato degrado (Fig. 4). Nel 1936, Pasquale Rotondi che nel 1939 avrebbe assunto il ruolo di Soprintendente alle Gallerie e alle Opere d'Arte delle Marche, elenca i principali monumenti di Arquata a cui era vicino per motivi familiari. Aveva, infatti, sposato la storica dell'arte Zea Bernardini, la cui madre era originaria di Arquata. L'elenco elaborato da Rotondi era diviso in quattro voci: le case, le chiese, le mura e i torrioni. Sulla Rocca, Rotondi scriveva "di epoca medievale sulla via omonima, con torre cubica a merlatura ghibellina e difesa piombante tra beccatelli (secolo XIII) con cortine verticali originariamente merlate (secoli XIII-XIV); torrione circolare munito di controscarpa speronata (secolo XV) e ampliamenti della fine del 1400"1. Il documento è importante per illuminare l'identità artistica di Arquata e per orientare le ricerche e l'auspicabile ricostruzione dopo le distruzioni del terremoto.

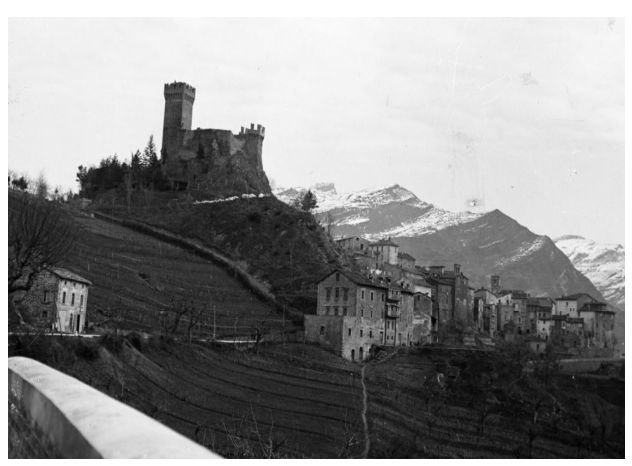

Fig. 4. Immagine della rocca nei primi anni del XX secolo. Fondo Corsini, Castelli 1920-1930, Fotografie, https://www.ankonet.eu/corsini/wp-content/gallery/ fondo-corsini/22.jpg.

\section{Le evidenze architettoniche}

Il piccolo fortilizio arquatano è stato definito una "realizzazione elegante ed equilibrata", risultato di numerosi interventi, soprattutto per assecondare i profondi mutamenti nelle tecniche di difesa (Cassi Ramelli, 1964, pp. 56-80). La struttura 
della Rocca è realizzata principalmente con blocchi di pietra arenaria locale. Sulla sommità delle torri è presente un aggetto eseguito in laterizio e travertino, idoneo alla difesa piombante, rifinito da una merlatura a coda di rondine, anch'essa in laterizio. L'impianto essenziale della Rocca si caratterizza per una cinta muraria di forma allungata, con due torri e alcuni resti di un terzo torrione. L'intera area è stata circondata da un parco che racchiude gli spazi di pertinenza della fortificazione e il percorso di collegamento con il borgo sottostante.

Gli edifici sono collegati tra loro tramite una recinzione in muratura con un camminamento di ronda. L'ingresso al recinto della fortezza era protetto dalla presenza di una bertesca lignea sostenuta da beccatelli in pietra, ancora visibili. L'intera fortificazione, oltre che dalla barriera muraria, era circondata da una palizzata che costituiva il primo elemento di difesa ad ostacolo degli assalitori.

Nel tessuto murario del recinto si trovano blocchi di pietra di notevoli dimensioni, probabilmente appartenute a una fase antica della struttura, a testimonianza dell'origine romana dell'insediamento. Durante il Medioevo, i blocchi di dimensioni più grandi venivano rilavorati, per ottenere conci lapidei più piccoli e facilmente trasportabili; questi andavano a comporre $\mathrm{i}$ muri in elevato, spesso affidandone la portanza a una doppia foderatura che conteneva il nucleo di materiale più scadente.

Il primo elemento edificato sul colle fu il torrione di pianta esagonale, alto circa 12 metri e situato sullo spigolo sud-est. Collegata al torrione esagonale era stata realizzata una cinta muraria, lunga circa $70 \mathrm{~m}$. Tra il XIV ed il XV secolo, era stato costruito il mastio, esposto a nord, destinato all'avvistamento e alla difesa estrema. La torre si elevava da una base quadrata il cui lato misurava circa $8 \mathrm{~m}$ e raggiungeva un'altezza di 24 $\mathrm{m}$. Alla sommità di questa torre si trovava la stanza quadrangolare, realizzata durante il restauro eseguito nei primi del XX secolo, in sostituzione di una volta a vela in muratura che proteggeva la campana di allarme della fortezza. La torre nord era collegata con la zona sud tramite una doppia cinta muraria dotata di camminamen- to di ronda, piombatoi e apparato aggettante. Il tutto delimitava uno spazio di circa $21 \times 24 \mathrm{~m}$ all'interno del quale dovevano essere collocate le costruzioni, per consentire agli armigeri di vivere arroccati all'interno del castello, soprattutto durante la lunga stagione invernale.

\section{Analisi degli interventi di restauro}

Nel corso dei secoli la Rocca è stata oggetto di vari interventi di restauro. Nel 1703 il terremoto dell'Aquila aveva causato danni alla struttura della Rocca, determinando la necessità di urgenti interventi di restauro ${ }^{2}$ (Galiè, Vecchioni, 2006, p. 84). Agli inizi del XX secolo, in occasione di un devastante terremoto fu realizzato un nuovo intervento ${ }^{3}$. La Rocca è stata interessata da un consistente intervento nei primi del '900 ad opera dell'Ufficio per la Conservazione dei Monumenti delle Marche e dell'Umbria, sotto la direzione di Giuseppe Sacconi ${ }^{4}$ che dimostra di voler aderire alla corrente del restauro storico, attraverso la ricomposizione delle antiche forme medievali, di cui possedeva chiari riferimenti lessicali (Figg. 5 e 6).

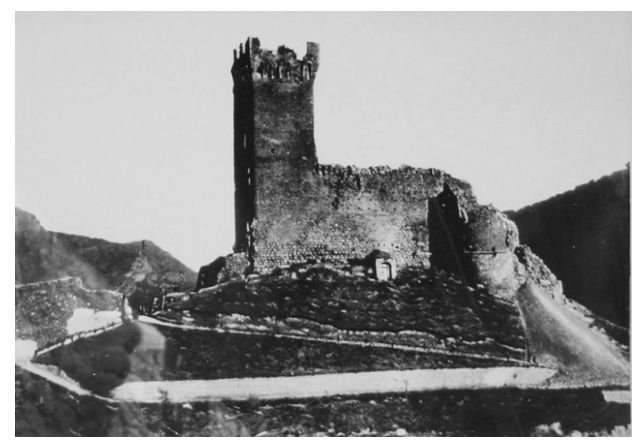

Fig. 5. La Rocca allo stato di rudere, prima degli interventi di restauro progettati da Giuseppe Sacconi nei primi del ' 900.

Nel 1966 ulteriori interventi conservativi hanno interessato la fortificazione arquatana. L'ultimo intervento di restauro è avvenuto alla metà degli anni '90; all'interno del recinto fortificato viene allestita una sala polifunzionale e organizzati alcuni spazi museali. Nel corso di uno scavo, eseguito all'interno della corte, nel 1992, era emerso uno stemma datato 1389 , appartenuto al guerriero Sinibaldo della famiglia Cancellieri di Pi- 
stoia, come si legge nell'iscrizione in caratteri gotici incisa alla base dello scudo. La presenza del reperto è legata alla consuetudine trecentesca di apporre sulle mura delle fortezze le insegne araldiche dei castellani che le amministravano.

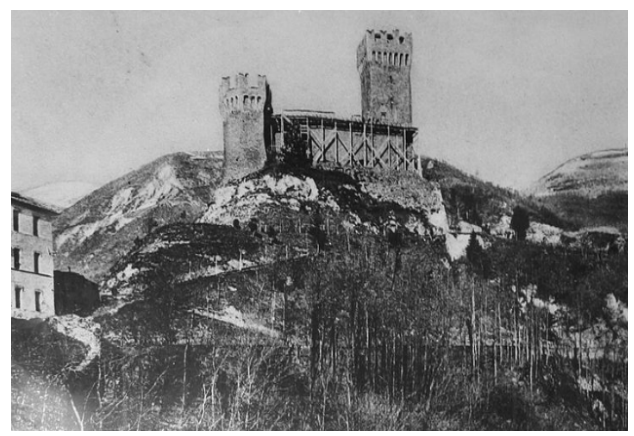

Fig. 6. Immagine dei lavori in corso di esecuzione, anno $1910 \mathrm{c}$.

\section{Gli eventi sismici e lo stato dei luoghi}

Arquata del Tronto è stata colpita dagli eventi simici 2016-2017 che hanno investito le aree interne delle Marche centro - meridionali. Dai dati reperibili dai resoconti più antichi (Baratta, 1901, p. 105), si può valutare come la zona sia ad elevata sismicità, risentendo sia dei terremoti che corrispondono alle faglie fra Marche e Umbria, sia quelli che hanno epicentri nella regione Abruzzo. Questi ripetuti terremoti hanno indotto nella popolazione differenti atteggiamenti per affrontare la catastrofe, con interventi che hanno ricostruito gli edifici crollati, con scarsa qualità materica e con una progettazione lontana dal rispetto della "regola dell'arte.

La fortezza di Arquata del Tronto, con la sua struttura massiva, ha resistito agli ultimi eventi sismici. Il particolare comportamento delle strutture di fortificazione alle azioni sismiche è stato studiato solo di recente, attraverso alcuni articoli che hanno individuato una serie di specificità, in relazione ad alcuni parametri di riferimento (Coïsson, Ferretti, Lenticchia, 2017, pp. 51395166). Si è tentato un approccio di tipo fenomenologico, che parte dal reale comportamento delle strutture, rilevabile in occasione dei terremoti, per valutarne i meccanismi di danno più tipici, riconducendolo ad alcune classi di comportamento (Coïsson, Lenticchia, 2017, pp. 2-7). Si richiamano, inoltre, alcuni studi compiuti sulla rocca roveresca di Senigallia, che hanno consentito di sottoporre il complesso fortificato ad una modellazione per simularne il comportamento sotto differenti azioni (Dall'Asta A., et al., 2019, pp. 86-98). Per quanto riguarda la Rocca di Arquata, dal sopralluogo effettuato nel febbraio 2017, si evince lo stato di danno che è da mettere in relazione con la tecnica costruttiva muratura a sacco: i due paramenti esterni non presentano elementi di connessione, pertanto la muratura risulta costituita da tre strati indipendenti e scollegati Nel prospetto ovest si è verificato il crollo di una porzione del paramento murario esterno, realizzato in blocchi squadrati di arenaria, per circa 1/3 del totale. L'angolata ovest-sud presenta una lesione ad andamento sub-verticale. In corrispondenza del prospetto sud, il distacco dell'angolata muraria è connotato da una profonda lesione. Sul prospetto est sono presenti ampie aree di caduta del paramento murario (Fig. 7).

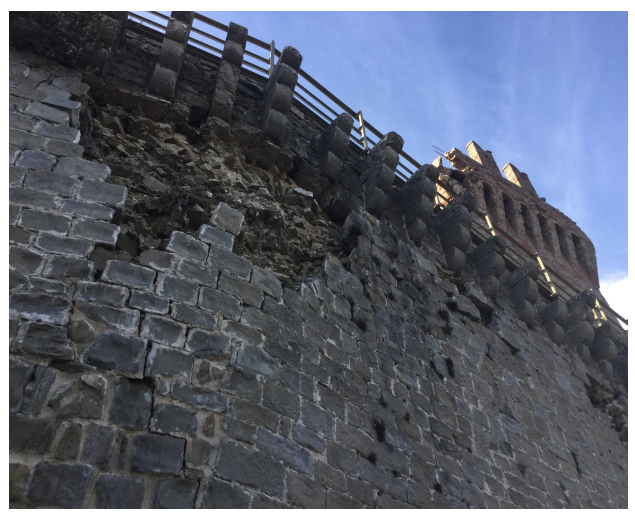

Fig. 7. Zona di caduta del paramento murario che lasciano scoperto il nucleo interno decoeso.

Alcune parti del paramento murario non crollato risultavano distaccate dal nucleo interno e con un meccanismo di rotazione innescato. La muratura in corrispondenza dell'attacco tra la torre femmina e la parete est della Rocca era in fase di distacco. Sulla sommità della torre femmina e della torre maschio erano crollate tutte le merlature. Il mastio è interessato da un quadro fessurativo caratterizzato da lesioni a $45^{\circ}$ su tutti i prospetti. In prossimità del portale di accesso al camminamento era evidente uno spostamento 
della muratura nell'ordine di circa $10 \mathrm{~cm}$. La copertura interna, aggiunta durante gli ultimi restauri, era in parte crollata a causa della caduta delle merlature (Fig. 8).
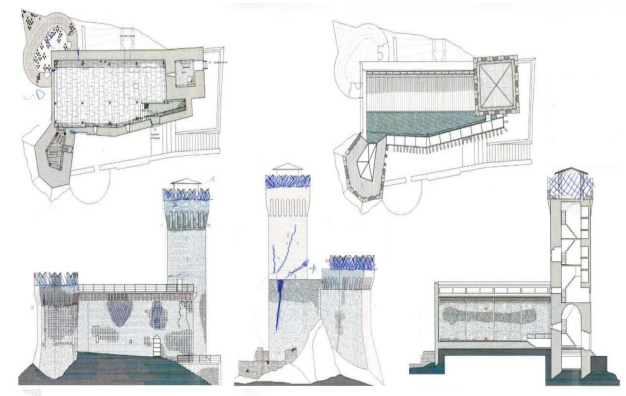

Fig. 8. Disegni illustrativi delle zone danneggiate, allegato alla Scheda GTS del 23/02/2017.

Sotto la supervisione della Soprintendenza Archeologia, Belle Arti e Paesaggio delle Marche sono stati eseguiti gli interventi di messa in sicurezza della Rocca (Fig. 9). L'intervento si è concentrato nei punti in cui si erano verificati i crolli più estesi. All'interno sono state eliminate le coperture sfondate e installati i ponteggi a tubo giunto sulle pareti perimetrali interne. La torre più alta è stata messa in sicurezza, con un doppio ordine di ponteggi a tubo e giunto e un nuovo rivestimento provvisorio realizzato con fodera in fibra, per recuperare solidità alla struttura.

Successivamente all'installazione dei ponteggi, si è proceduto all'applicazione di una serie di tiranti metallici. Una coppia di travi ed una serie di barre filettate e dotate di piastre di fissaggio hanno composto un dispositivo per tenere uniti fra loro i due ponteggi, che ingabbiano la muratura. Un semplice sistema definito "a sandwich", ma che ha avuto l'importante funzione di collegare i due ponteggi, comprimendoli contro le pareti e bloccandole al loro interno. Conclusa la fase della messa in sicurezza sono stati avviati gli studi propedeutici all'esecuzione dei lavori di consolidamento con miglioramento sismico, per ripristinarne la funzionalità, conservando la principale funzione di museo di sé stesso e di piccola realtà espositiva del territorio arquatano, riferimento della comunità locale arquatana, protetta dalla presenza della rocca, posta nella parte più elevata del borgo.

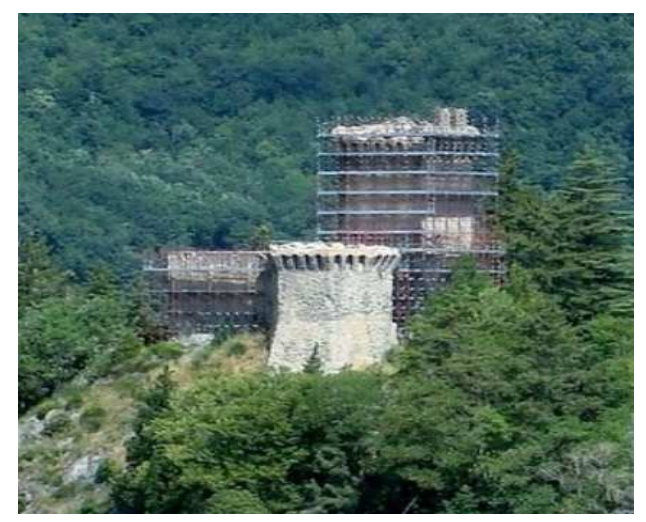

Fig. 9. Vista della Rocca di Arquata durante i lavori di messa in sicurezza.

\section{Conclusioni}

L'architettura fortificata costituisce un patrimonio molto rappresentativo di luoghi, storie, comunità del territorio marchigiano, ancora poco indagato in quanto gli studi hanno riguardato solo gli episodi architettonici più rilevanti, mentre i beni minori, dispersi nelle aree interne sono pressoché sconosciuti. Deve, quindi, essere ancora scritta la storia delle piccole realtà castellari, soprattutto quella delle fortificazioni che insistono nelle aree colpite dal sisma 2016-17. La rocca di Arquata del Tronto, ingabbiata dall'impalcatura di sicurezza, rappresenta nonostante le problematiche occorse a seguito degli eventi sismici, un simbolo di riferimento per la comunità arquatana. Per questo motivo, occorre che l'intervento di restauro assuma un ruolo significativo per l'intero territorio, sperimentando le più aggiornate tecniche nel campo del restauro.

\section{Note}

${ }^{1}$ https://www.giannellachannel.info/censimentomonumenti-arquata-del-tronto-prima-terremotorotondil (2 October 2019).

${ }^{2}$ Archivio di Stato di Ancona, Soprintendenza ai Monumenti per le Marche, Amministrazione, Rendiconti, 1899-1904, Rocca d'Arquata, 1903-1904, Busta 183.

${ }^{3}$ Archivio di Stato di Ancona, Soprintendenza ai Monumenti per le Marche, Tutela, Arquata del Tronto: Rocca, 1894-1910, Restauri, Busta 94. 
${ }^{4}$ Archivio di Stato di Ancona, Soprintendenza ai Monumenti per le Marche, Amministrazione, Ricognizio- ne restauro monumenti, 1920-1921, Busta 15, Fascicolo 35 .

\section{Bibliography}

Amoni, D. (1999). Castelli, fortezze e rocche dell'Umbria, Quattroemme, Perugia.

Andreantonelli, S. (2007). Storia di Ascoli, Gagliardi Ed., Ascoli Piceno.

Antonelli, M. (1994). “Chiese, abbazie, fortificazioni”, in Guide al Piceno, Sibillini, Maroni Ed., Ripatransone.

Baratta, M. (1901). I terremoti d'Italia: Saggio di storia, geografia e bibliografia sismica italiana, Fratelli Bocca Ed., Torino.

Bucciarelli, A. (1982). Dossier Arquatano, Grafiche D’Auria, Ascoli Piceno.

Carfagna, B. (1996). Rocche e castelli dell'ascolano, Edizione La Sfinge Malaspina, Ascoli Piceno.

Cassi Ramelli, A. (1964). Dalle caverne ai rifugi blindati. Trenta secoli di architettura militare, Nuova accademia, Siena.

Coïsson, E.; Ferretti, D.; Lenticchia, E. (2017). "Analysis of damage mechanisms suffered by Italian fortified buildings hit by earthquakes in the last 40 years", Bulletin of Earthquake Engineering, 15, 12, pp. 5139-5166.

Coïsson, E.; Lenticchia, E. (2017). "La fragilità delle fortezze. Nuovi strumenti per proteggere il patrimonio dell'architettura fortificata italiana dai terremoti”, ANANKE Speciale GEORES 2017, pp. 2-7.

Dall'Asta, A.; Leoni, G.; Meschini, A.; Petrucci, E.; Zona, A. (2019). "Integrated approach for seismic vulnerability analysis of historic massive defensive structures", Journal of Cultural Heritage, 35, pp. 86-98.

Fabiani, G. (1950). Ascoli nel Quattrocento, Collana di pubblicazioni storiche ascolane, Società Tipolitografica Editrice, Ascoli Piceno, vol. II.

Galiè, N.; Vecchioni, G. (2006). Arquata del Tronto: il Comune dei due Parchi Nazionali, Stampa D'Auria Industrie Grafiche, Ascoli Piceno.

Girolami, L. (1987). "La Rocca di Arquata”, Flash Ascoli - mensile di vita Picena, 107.

Luzi, E. (1889). Compendio di storia ascolana, Emidio Cesari Editore, Ascoli Piceno, pp. 106, 123-126.

Mauro, M. (1992). Castelli, rocche, torri, cinte fortificate delle Marche, Istituto italiano dei Castelli Sezione Marche, Ancona, vol. I.

Meschini, A.; Petrucci, E. (2018). "Rappresentazione sincronica e ricostruzioni diacroniche della Rocca di Senigallia. Un approccio di conoscenza integrato", in FORTMED 2018. Defensive Architecture of the Mediterranean, International Conference on Modern Age Fortification of the Mediterranean Coast, vol. IX, pp.1013-1020.

Sacconi G. (1903). Relazione dell'Ufficio Regionale per la conservazione dei Monumenti delle Marche e dell'Umbria, Tipografia Guerriero Guerra, Perugia.

Salvi, A. (2010). Iscrizioni medievali nel territorio ascolano. Documenti epigrafici con relative note storiche, Istituto superiore di studi medievali Cecco d'Ascoli, Ascoli Piceno.

Santis, A. De. (1999). Ascoli nel Trecento, Collana di Pubblicazioni Storiche Ascolane, Grafiche D’Auria, Ascoli Piceno, vol. II.

Settia, A. (2017). Castelli Medievali, Il Mulino, Bologna. 